\title{
Age and Sex Effects on White Matter Tracts in Psychosis from Adolescence through Middle Adulthood
}

\author{
Andrew Schwehm', Delbert G Robinson ${ }^{2,3,4,5}$, Juan A Gallego ${ }^{2,3,4,5}$, Katherine H Karlsgodt ${ }^{2,3,4,5}$, \\ Toshikazu Ikuta ${ }^{6}$, Bart D Peters ${ }^{2,3}$, Anil K Malhotra ${ }^{2,3,4,5}$ and Philip R Szeszko*,7,8 \\ 'Department of Psychology, St John's University, Jamaica, NY, USA; ${ }^{2}$ Center for Psychiatric Neuroscience, The Feinstein Institute for Medical \\ Research, Manhasset, NY, USA; ${ }^{3}$ Division of Psychiatry Research, Zucker Hillside Hospital, North Shore-LIl Health System, Glen Oaks, NY, USA; \\ ${ }^{4}$ Department of Psychiatry, Hofstra North Shore - LIJ School of Medicine, Hempstead, NY, USA; ${ }^{5}$ Department of Molecular Medicine, Hofstra \\ North Shore - LIJ School of Medicine, Hempstead, NY, USA; 'Department of Communication Sciences and Disorders, School of Applied Sciences, \\ University of Mississippi, Oxford, MS, USA; ${ }^{7}$ James J. Peters Veterans Affairs Medical Center, Bronx, NY, USA; ${ }^{8}$ Department of Psychiatry, Icahn \\ School of Medicine at Mount Sinai, New York, NY, USA
}

There is controversy regarding specificity of white matter abnormalities in psychosis, their deviation from healthy aging, and the influence of sex on these measures. We used diffusion tensor imaging to characterize putative white matter microstructure in 224 patients with psychosis and healthy volunteers across the age range of 15-64 years. Sixty-five younger (age <30 years; 47M/I 8F) patients with psychosis (all experiencing a first episode of illness) and 48 older (age $\geqslant 30$ years; 30M/I 8F) patients were age-matched to younger and older healthy volunteer groups ( $N=63(40 \mathrm{M} / 23 \mathrm{~F})$ and $N=48$ (29M/I9F), respectively). The trajectories of two inter-hemispheric (splenium and genu), two projection (cortico-pontine and anterior thalamic), and five bilateral association (inferior fronto-occipital, inferior longitudinal, superior longitudinal, cingulum, and uncinate) tracts were quantified using tractography to derive measures of fractional anisotropy and mean, axial, and radial diffusivity. Fractional anisotropy was significantly lower in the inferior longitudinal fasciculus and superior longitudinal fasciculus in all patients compared with all healthy volunteers, with comparable effect sizes observed in both the younger and older patients compared with their respective healthy volunteer groups. Moreover, age-associated differences in fractional anisotropy within these tracts were comparable between groups across the age span. In addition, female patients had significantly lower fractional anisotropy across all tracts compared with female controls regardless of age. Our findings demonstrate comparable putative white matter abnormalities in two independent samples of patients with psychosis and argue against their progression in patients. These data further highlight the novel and potentially underappreciated role of sex in understanding white matter dysfunction in the neurobiology of psychosis.

Neuropsychopharmacology (2016) 4I, 2473-2480; do: I 0.1038/npp.20 I6.47; published online I8 May 2016

\section{INTRODUCTION}

Diffusion tensor imaging (DTI) is an important tool for characterizing neuroanatomical connectivity deficits in the pathophysiology of schizophrenia and associated psychotic disorders (Kubicki et al, 2008; White et al, 2008). Initial DTI studies reported widespread white matter abnormalities throughout the brain (Lim et al, 1999) and frontal lobes (Buchsbaum et al, 1998) of patients with chronic schizophrenia. Subsequent studies identified white matter abnormalities encompassing much of the brain white matter but consistent with models of frontotemporal dysfunction (eg, see Ellison-Wright and Bullmore, 2009 for a metaanalysis).

\footnotetext{
*Correspondence: Dr PR Szeszko, James J. Peters Veterans Affairs Medical Center, Mental Health Care Center, I30W Kingsbridge Road, Bronx, NY 10468, USA, Tel: + I 7I8 5849000 ext 5869, Fax: + I 7I8 74 I 4703, E-mail: Philip.Szeszko@va.gov

Received 2 September 2015; revised 24 March 2016; accepted 3 April 2016; accepted article preview online 12 April 2015
}

Fewer studies have investigated white matter using DTI in patients with psychosis early in the course of illness. Evidence for white matter abnormalities in antipsychotic drug-naive patients with psychosis (Cheung et al, 2008; Szeszko et al, 2005) suggests that they are not an artifact of drug treatment or illness progression. A recent meta-analysis examining eight studies in first-episode schizophrenia reported lower fractional anisotropy (FA) in multiple regions, including the right deep frontal and left deep temporal lobes (Yao et al, 2013). Some first-episode studies may have a number of limitations, however, including assessment of a few brain regions, inconsistency regarding methods, and small samples. For example, of the 10 firstepisode studies reviewed by Fitzsimmons et al (2013), all but one had sample sizes of $\leqslant 20$.

DTI studies comparing younger and older patients with psychosis may clarify the relationship between illness progression and white matter. Several studies reported that white matter abnormalities in first-episode patients are less robust compared with chronic patients (Friedman et al, 2008; 
White et al, 2011); however, these studies focused on limited and/or gross regions of interest and have not used tractography. Moreover, little is known regarding how deviations from the normal developmental trajectory may contribute to psychosis (Peters and Karlsgodt, 2014). Although several studies reported evidence for accelerated age-associated white matter FA differences in schizophrenia (Kochunov et al, 2013; Rosenberger et al, 2008; Schneiderman et al, 2011; Wright et al, 2014), others have not (Voineskos et al, 2010). Possible limitations of prior work include the limited age range investigated, lack of firstepisode patients and/or sole investigation of FA.

Sex is an important consideration in neuroimaging studies of psychosis given that it may influence age at onset, illness progress, clinical manifestation, and antipsychotic treatment response (Abel et al, 2010; Goldstein et al 2013). Some studies reported sex-specific brain abnormalities linked to resting-state functional (Lei et al, 2015) and structural (Trzesniak et al, 2011) abnormalities. In addition, a prior study investigating structure-function relations in schizophrenia reported that worse executive and motor functioning were associated with less anterior hippocampal volume in male but not in female patients (Szeszko et al, 2002). Few studies, however, have specifically investigated the role of sex in mediating brain white matter abnormalities in psychosis using DTI. Some data implicate sex steroid effects on the white matter in animal models of schizophrenia, with estrogen providing a protective effect (Gogos et al, 2012) through mediation of BDNF expression and activity (Wu et al, 2013). Other data suggest a pattern of sexdependent white matter geometric deviations consistent with abnormal 'torque' (Savadjiev et al, 2014) and that age-related sex differences in white matter may be related to axonal radial growth outpacing concomitant increases in myelin thickness (Paus and Toro, 2009).

We investigated nine white matter tracts using probabilistic tractography in large cohorts of younger $(N=65)$ and older $(N=48)$ patients with psychosis compared with ageand sex-matched healthy volunteer groups $(N=63$ and $N=48$, respectively) using four indices of putative white matter microstructure (ie, FA, axial diffusivity (AD), radial diffusivity (RD), and mean diffusivity (MD)) and the potential role of sex. We hypothesized that younger patients would have significant, but less widespread, white matter abnormalities compared with older patients. An additional study goal was to examine age-associated differences in putative white matter microstructure from adolescence through middle adulthood in patients with psychosis compared with healthy volunteers.

\section{MATERIALS AND METHODS}

\section{Subjects}

Sixty-five younger (age <30) psychosis patients were recruited from admissions to the inpatient service at The Zucker Hillside Hospital as part of their participation in an NIMH-funded double-blind, randomized, controlled trial comparing aripiprazole with risperidone. Prior antipsychotic medication usage was recorded for patients prior to the MR imaging exam; this was limited to 2 weeks for clinical trial eligibility. Patients were not allowed to receive antidepressants or mood stabilizers as part of the clinical trial. Cogentin and/or propranolol were prescribed for extrapyramidal symptoms as required during the trial. Benzodiazepines (lorazepam) were the only class of medications allowed for antipsychotic-naive patients prior to the MR imaging scan.

All younger patients were experiencing a first episode of psychosis and received a physical exam and laboratory screening to rule out medical causes for this initial episode. Patients had a mean (SD) of 9.6 (7.2) days of antipsychotic treatment prior to the $\mathrm{MR}$ imaging exam, excluding 22 patients who were antipsychotic drug-naive at the time of the scan. Mean age at first psychotic symptoms was 18.7 ( $\mathrm{SD}=3.6)$. In addition, 48 older (age $\geqslant 30$ years) patients were recruited at our facility and were receiving the following antipsychotics at the time of the scan (numbers in parentheses): aripiprazole (12), risperidone (7), olanzapine (5), quetiapine (12), ziprasidone (3), haloperidol (4), paliperidone (1), fluphenazine (5), and clozapine (10). Medication information was unavailable for two patients. We used age 30 years as the cutoff between younger and older patients with psychosis based on our prior study indicating that the majority of white matter tracts have achieved maximum FA values by this age (Peters et al, 2014).

All patient diagnoses were based on the SCID for Axis I DSM-IV Disorders supplemented by information from clinicians and, when available, family members. Diagnoses for younger patients included schizophrenia $(n=41)$, schizoaffective disorder $(n=1)$, schizophreniform disorder $(n=16)$, or psychosis NOS $(n=7)$. Older patients with psychosis met DSM-IV criteria for schizophrenia $(n=38)$, schizoaffective disorder $(n=8)$, and psychosis NOS $(n=2)$. Thirty-one younger patients with psychosis and 26 older patients with psychosis met DSM-IV criteria for having a lifetime history of any substance use diagnosis.

Two separate cohorts of healthy volunteers $(N=63$; age $<30$ years and $N=48$; age $\geqslant 30$ years) were recruited from advertisements posted on websites and by word of mouth to match the demographic distributions of younger and older patients. Exclusion criteria for healthy volunteers included the denial of any lifetime history of a major mood or psychotic disorder as determined by clinical interview using the SCID-NP.

Exclusion criteria for all participants included: (a) MRI contraindications; (b) significant medical illness, including Gilles de la Tourette's, Huntington's disease, Parkinson's disease, encephalitis, strokes, aneurysms, tumors, CNS infections, degenerative brain diseases, cognitive disorders (ie, amnesia, dementia, and delirium), and history of cancer; (c) prior psychosurgery; (d) DSM-IV diagnosis of Tourette's syndrome, developmental disorders, autism, and neurological conditions; (e) DSM-IV mental retardation; (f) stroke; and (g) pregnancy. The study was approved by the NSLIJ IRB. Written informed consent was obtained from all individuals and from a parent or legal guardian in the case of minors. Written assent was obtained from all minors.

\section{Magnetic Resonance (MR) Imaging Procedures}

DTI exams were conducted at the North Shore Medical Center on a GE $3 \mathrm{~T}$ system. We minimized movement by stabilizing the head prior to scanning. A total of 36 DTI 


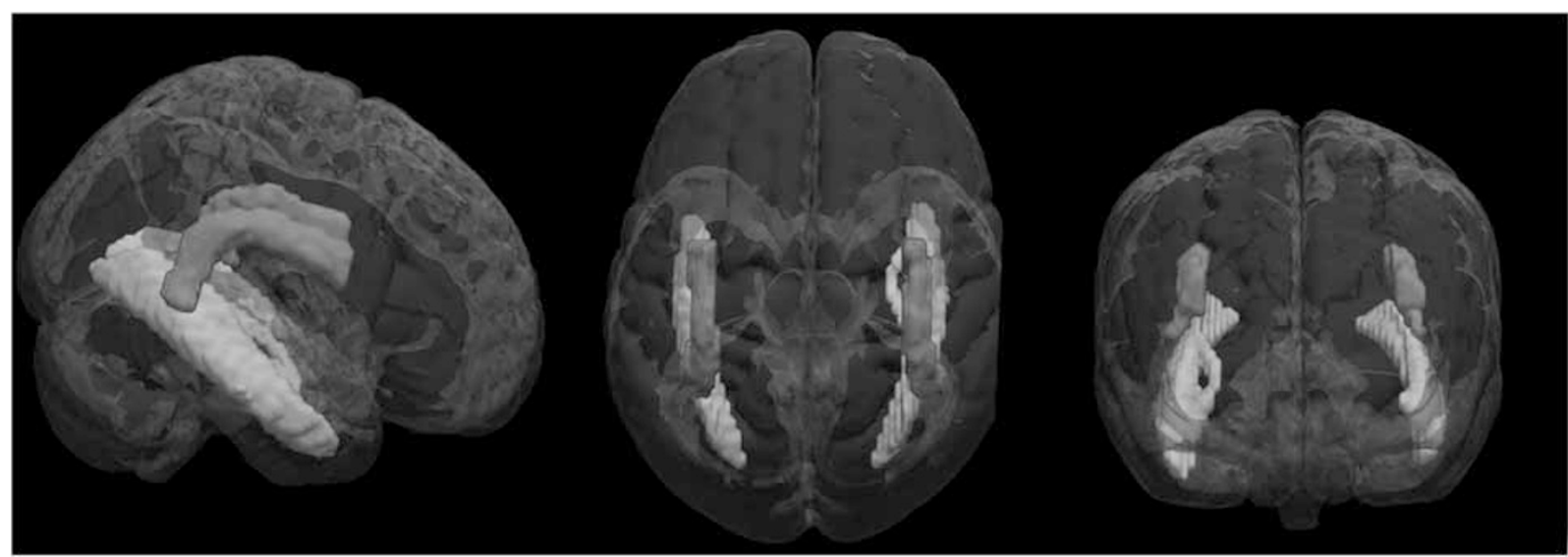

Figure I Illustration of the superior longitudinal fasciculus (red) and inferior longitudinal fasciculus (blue) assessed using probabilistic tractography. A full color version of this figure is available at the Neuropsychopharmacology journal online.

volumes were obtained from each subject that included 31 volumes with diffusion gradients applied along 31 nonparallel directions with $b=1000 \mathrm{~s} / \mathrm{mm}^{2}$ and, and 5 volumes without diffusion weighting $(b=0)$. Each volume consisted of 51 contiguous $2.5-\mathrm{mm}$ axial slices acquired parallel to the anterior-posterior (AC-PC) commissural line using a ramp sampled, spin-echo, single shot echo-planar imaging method $(\mathrm{TR}=14000 \mathrm{~ms}, \mathrm{TE}=\mathrm{min}, \quad$ matrix $=128 \times 128$, $\mathrm{FOV}=240 \mathrm{~mm})$.

\section{Image Processing}

Image processing was conducted using the Functional Magnetic Resonance Imaging of the Brain Software Library (FSL; Oxford, UK; http://fsl.fmrib.ox.ac.uk/fsl). Eddy-current-induced distortions and head motion were corrected through affine registration of the 31 diffusion volumes to the first b0 volume using the FSL's Linear Registration Tool (Jenkinson et al, 2012). The $b$-vector table (ie, gradient directions) for each participant was adjusted according to the rotation parameters of this linear correction. Non-brain tissue was removed using the FSL's Brain Extraction Tool. Measures were calculated at each voxel of the brain by fitting a diffusion tensor model to the raw diffusion data using weighted least squares in FSL's Diffusion Toolbox.

\section{Probabilistic Tractography}

The probable trajectories of two inter-hemispheric tracts (splenium and genu of corpus callosum), two projection tracts (cortico-pontine and anterior thalamic radiation), and five bilateral association tracts (inferior fronto-occipital fasciculus, inferior longitudinal fasciculus and superior longitudinal fasciculus, cingulum, and uncinate fasciculus) were traced using previously published methods (Figure 1; Peters et al, 2014). Within-voxel probability density functions of the principal diffusion direction were estimated using Markov Chain Monte Carlo sampling in the FSL's BEDPOSTX tool (Behrens et al, 2003). A spatial probability density function was then estimated across voxels based on these local probability density functions using the FSL's
PROBTRACKX tool, in which 5000 samples were taken for each input voxel with a 0.2 curvature threshold, $0.5-\mathrm{mm}$ step length, and 2000 steps per sample.

For each tract, seed masks, way-points, termination, and exclusion masks were defined on the MNI152 T1 1-mm template, using the FMRIB58 FA template as a DTI-specific reference. Masks were normalized to each subjects' diffusion space using FLIRT, inverting the affine parameters obtained by co-registering the first b0 volume to the MNI152 1-mm T1 brain. The resulting tracts were thresholded at a normalized probability value (available for each tract in the Supplementary Material of Peters et al, 2014) and visually inspected to confirm successful tracing in each individual. Normal probability values indicate the weighting assigned to each tract to ensure that the most likely tracts were included in the measurement. Successful tracing was defined as the program generating the tract and following visual inspection in all planes by an individual trained in neuroanatomy for consistency with standard neuroanatomical atlases. Masks are available upon request. We did not reject any tracts in this study that were included in any of our previous studies.

\section{Statistical Analysis}

We used repeated-measures ANOVA with group (patients $v s$ healthy volunteers), age group (younger $v s$ older), and sex as between-subjects factors. White matter tract (splenium of corpus callosum, genu of corpus callosum, cortico-pontine tract, anterior thalamic radiation, inferior fronto-occipital fasciculus, inferior longitudinal fasciculus, superior longitudinal fasciculus, cingulum, and uncinate fasciculus) served as a within-subjects factor in four separate analyses investigating the white matter measures. The GreenhouseGeisser correction was used in each analysis because Mauchly's test of sphericity was significant. We averaged right and left hemisphere measures to reduce Type-I error given the lack of significant group $\times$ hemisphere interactions. Effect size measures are represented as eta-squared, which can be interpreted as small $(\sim 0.02)$, medium $(\sim 0.13)$, and large ( 0.25). Pearson's product moment correlations were used to investigate the relationship between age and white matter measures in tracts that differed significantly between 
Table I Sample Demographics

\begin{tabular}{|c|c|c|c|c|c|c|c|c|}
\hline & \multicolumn{2}{|c|}{ Younger controls } & \multicolumn{2}{|c|}{ Younger patients } & \multicolumn{2}{|c|}{ Older controls } & \multicolumn{2}{|c|}{ Older patients } \\
\hline & $\begin{array}{c}\text { Males } \\
(N=40)\end{array}$ & $\begin{array}{l}\text { Females } \\
(N=23)\end{array}$ & $\begin{array}{c}\text { Males } \\
(N=47)\end{array}$ & $\begin{array}{l}\text { Females } \\
(N=18)\end{array}$ & $\begin{array}{c}\text { Males } \\
(N=29)\end{array}$ & $\begin{array}{l}\text { Females } \\
(N=19)\end{array}$ & $\begin{array}{c}\text { Males } \\
(N=\mathbf{3 0})\end{array}$ & $\begin{array}{c}\text { Females } \\
(N=18)\end{array}$ \\
\hline Age (years) & $20.1(4.1)$ & $20.7(2.5)$ & $21.1(3.3)$ & $19.9(4.0)$ & $43.6(8.8)$ & $45.7(9.1)$ & $45.0(8.7)$ & $46.2(9.7)$ \\
\hline Laterality quotient & $0.7 \mid(44)$ & $0.69(0.54)$ & $0.65(0.45)$ & $0.73(0.54)$ & $0.76(0.53)$ & $0.86(0.43)$ & $0.55(0.7 I)$ & $0.97(0.06)$ \\
\hline
\end{tabular}

Notes: Data are presented as mean \pm SD in parentheses, unless otherwise indicated. Younger is defined as $<30$ years, whereas older is defined as age $\geqslant 30$ years.

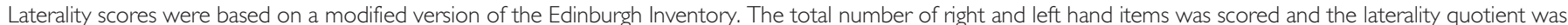
computed: (Total $\mathrm{R}-$ Total $\mathrm{L}) /($ Total $\mathrm{R}+$ Total $\mathrm{L}$ ) yielding a range from $+\mathrm{I} .00$ (totally dextral) to - I.00 (totally nondextral).

groups. We tested the difference between groups using Fisher's $r$-to- $z$ transformations. In addition, we tested the group-by-age (and age ${ }^{2}$ ) interaction for white matter tracts that differed significantly between groups to identify possible group differences in age-associated white matter differences across the age span. All analyses were two-tailed with alpha set to 0.05 .

\section{RESULTS}

Demographic and clinical characteristics are provided in Table 1. The younger and older patient cohorts did not differ significantly from their respective healthy volunteer cohorts in distributions of age and handedness either when analyzed separately by sex or not $(p s>0.05)$. Education differed significantly between younger female patients compared with younger female healthy volunteers and between older male patients and older female patients compared with their matched older healthy volunteer groups, respectively. Mean (SD) Brief Psychiatric Rating Scale score was $43.8(\mathrm{SD}=8.8)$ for younger patients and $32.1(\mathrm{SD}=7.8)$ for older patients. Average illness duration for first-episode and chronic psychosis patients was 1.9 years $(\mathrm{SD}=2.3)$ and 24.7 years $(\mathrm{SD}=10.8)$, respectively. Mean values for the diffusion measures are provided in Table 2 for descriptive purposes only.

There were significant main effects of group for FA, RD, and MD. Overall, compared with healthy volunteers, patients had lower FA $(\mathrm{F}=9.79, \mathrm{df}=1,213, p=0.002)$, higher $\mathrm{MD}$ $(\mathrm{F}=6.82, \mathrm{df}=1,213 p=0.01)$, and higher $\mathrm{RD}(\mathrm{F}=10.46$, $\mathrm{df}=1,213, p=0.001)$ across all white matter tracts. There was a significant $(\mathrm{F}=2.20, \mathrm{df}=5.57, p=0.045)$ group $\times$ tract interaction for $\mathrm{FA}$, indicating that patients differed from healthy volunteers in some tracts but not in others. Specifically, FA was significantly lower in patients compared with healthy volunteers in the inferior longitudinal fasciculus $(p=0.004$; see Figure 1) and superior longitudinal fasciculus $(p=0.022$; see Figure 1$)$. We examined whether substance use might be influencing these findings, but there were no significant FA differences between patients with and without a substance use diagnosis in these tracts.

Post-hoc analyses indicated significant main effects of group for $\mathrm{FA}$ in the superior longitudinal fasciculus both in the younger $(\mathrm{F}=7.53 \mathrm{df}=1,123, p=0.007$; effect size $=0.058)$ and older $(\mathrm{F}=4.16, \mathrm{df}=1,92, p=0.044$; effect size $=0.043)$ patient groups compared with their respective healthy volunteer groups. Similarly, the main effect of group was significant in the younger $(\mathrm{F}=6.10, \mathrm{df}=1,124$, $p=0.015$; effect size $=0.047)$ and older $(\mathrm{F}=7.05, \mathrm{df}=1,92$, $p=0.009$; effect size $=0.071)$ patient groups compared with their respective healthy volunteer groups for the inferior longitudinal fasciculus. No group-by-tract interactions were significant for $\mathrm{RD}, \mathrm{AD}$, and $\mathrm{MD}$. In addition, none of the group-by-tract-by-sex interactions were statistically significant for any of the white matter measures.

\section{Age Effects}

Overall, there was a significant main effect of age group such that compared with younger individuals, older individuals had lower FA $(\mathrm{F}=20.00, \mathrm{df}=1,213, p<0.001)$, higher $\mathrm{MD}(\mathrm{F}=4.70, \mathrm{df}=1,213, p=0.031)$, and higher $\mathrm{RD}$ $(\mathrm{F}=14.66, \mathrm{df}=1,213, p<0.001)$ across the white matter tracts. Age correlated significantly and inversely with FA in the inferior longitudinal fasciculus in patients $(r=-0.27$, $\mathrm{df}=113, p=0.004)$ and healthy volunteers $(r=-0.32$, $\mathrm{df}=111, p=0.001)$. Age correlated inversely but not significantly with FA in the superior longitudinal fasciculus in patients $(r=-0.14, \mathrm{df}=111, p>0.05)$ and healthy volunteers $(r=-0.18, \mathrm{df}=110, p>0.05)$. The difference between correlation coefficients was not significantly different between patients and healthy volunteers either in the inferior longitudinal fasciculus $(z=-0.4$; see Figure 2$)$ or superior longitudinal fasciculus $(z=-0.32$; see Figure 3$)$. We further tested the age $\times$ group and the age ${ }^{2} \times$ group interactions for FA within the superior longitudinal fasciculus and inferior longitudinal fasciculus. None of the age group-bygroup interactions were statistically significant for any of the white matter measures. These findings were confirmed in the subgroup of patients without a substance use diagnosis and were comparable between patients and healthy volunteers when analyzed separately by sex.

\section{Sex Effects}

There was a significant main effect of sex such that males had higher FA compared with females $(\mathrm{F}=18.62$, $\mathrm{df}=1,213$, $p<0.001)$ and females had higher $\mathrm{RD}$ than males $(\mathrm{F}=8.09$, $\mathrm{df}=1,213, p=0.005)$. There was a significant group-by-sex interaction for $\mathrm{FA}(\mathrm{F}=15.88, \mathrm{df}=1,213, p<0.001), \mathrm{MD}$ $(\mathrm{F}=15.88, \mathrm{df}=1,213, p<0.001)$, and $\mathrm{RD}(\mathrm{F}=8.57, \mathrm{df}=1$, 213, $p=0.004$ ) such that female patients had significantly lower FA across all the white matter tracts compared with 
Table 2 Mean Tractography Values

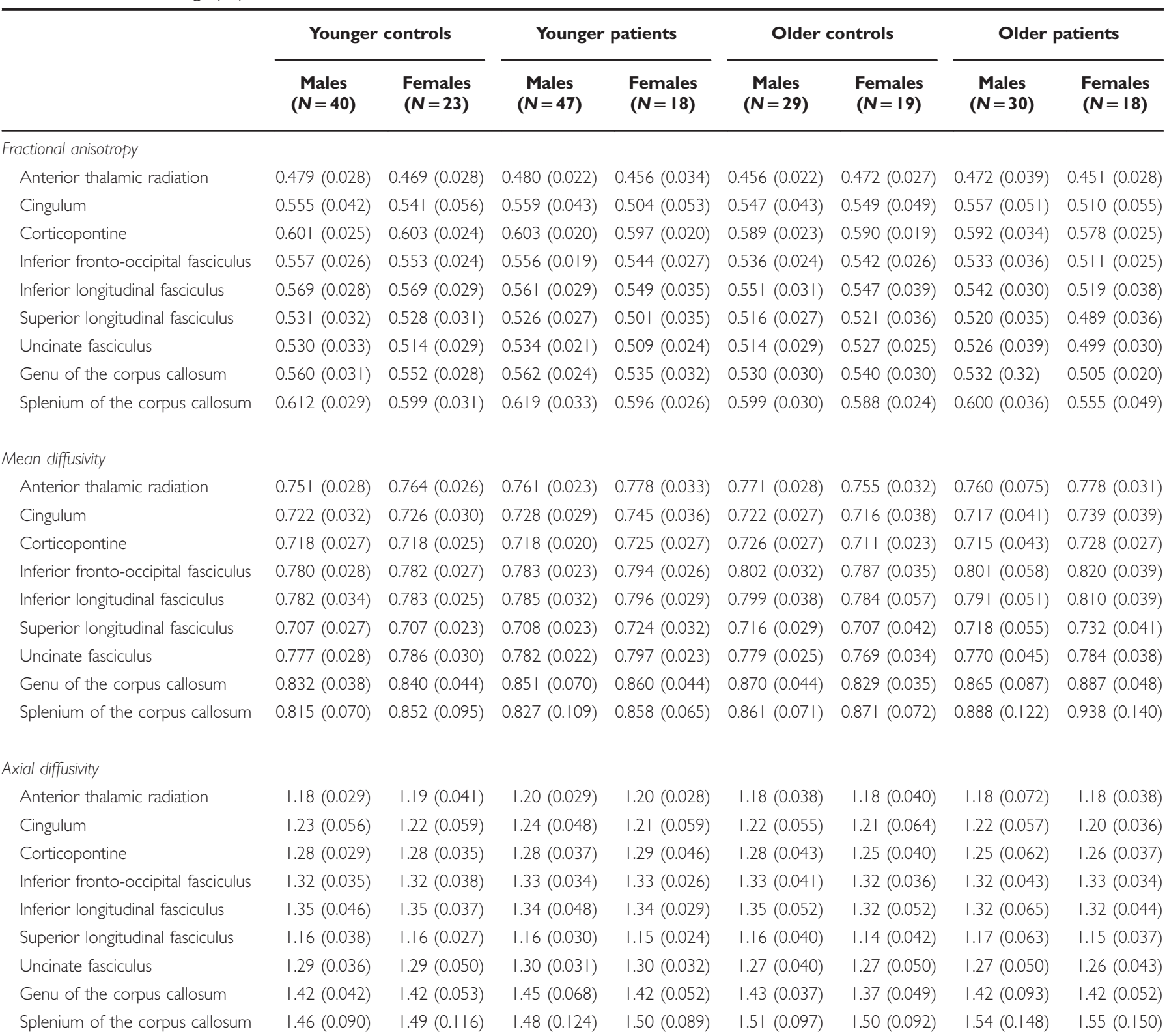

\section{Radial diffusivity}

Anterior thalamic radiation

Cingulum

Corticopontine

Inferior fronto-occipital fasciculus Inferior longitudinal fasciculus

Superior longitudinal fasciculus

Uncinate fasciculus

Genu of the corpus callosum

Splenium of the corpus callosum

$\begin{array}{lllllllll}0.536(0.033) & 0.550(0.029) & 0.542(0.026) & 0.569(0.043) & 0.564(0.029) & 0.544(0.035) & 0.551(0.078) & 0.574(0.034) \\ 0.468(0.042) & 0.480(0.052) & 0.470(0.042) & 0.515(0.050) & 0.474(0.039) & 0.515(0.050) & 0.466(0.053) & 0.510(0.058) \\ 0.438(0.032) & 0.435(0.027) & 0.436(0.021) & 0.442(0.026) & 0.451(0.027) & 0.441(0.022) & 0.446(0.042) & 0.460(0.030) \\ 0.507(0.033) & 0.511(0.030) & 0.510(0.025) & 0.525(0.035) & 0.537(0.035) & 0.523(0.035) & 0.54 I(0.063) & 0.568(0.042) \\ 0.500(0.037) & 0.500(0.033) & 0.508(0.037) & 0.522(0.04 I) & 0.522(0.043) & 0.517(0.065) & 0.526(0.050) & 0.553(0.048) \\ 0.480(0.034) & 0.482(0.032) & 0.484(0.029) & 0.51 I(0.042) & 0.493(0.034) & 0.488(0.049) & 0.494(0.057) & 0.523(0.048) \\ 0.521(0.036) & 0.536(0.031) & 0.520(0.026) & 0.546(0.028) & 0.531(0.031) & 0.517(0.033) & 0.521(0.050) & 0.546(0.042) \\ 0.541(0.045) & 0.550(0.046) & 0.554(0.074) & 0.578(0.048) & 0.593(0.053) & 0.555(0.040) & 0.588(0.086) & 0.622(0.048) \\ 0.493(0.066) & 0.531(0.089) & 0.497(0.105) & 0.537(0.058) & 0.538(0.065) & 0.558(0.067) & 0.564(0.113) & 0.632(0.142)\end{array}$

Notes: Data are presented for descriptive purposes only (see text for a description of the statistical analysis plan). SDs are in parentheses. FA values are dimensionless and multiplied by 1000. Axial, radial, and mean diffusivity units are $10^{-3} \mathrm{~mm}^{2} / \mathrm{s}$. 


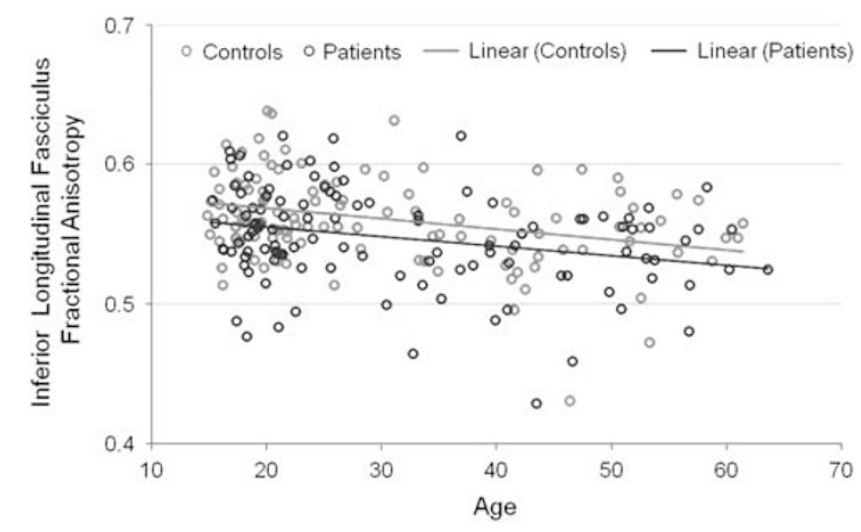

Figure 2 Linear scatterplot of age with fractional anisotropy in the inferior longitudinal fasciculus. Note: The interactions of age and age ${ }^{2}$ (not illustrated) with group were not statistically significant.

healthy female volunteers. Similarly, for both MD and RD this effect was driven by female patients having significantly higher values overall across the tracts compared with healthy female volunteers. Post-hoc analyses investigating sex differences for significant tracts in the omnibus test indicated that female patients had significantly lower FA in the superior longitudinal fasciculus compared with male patients in the younger age group $(t=3.07, \mathrm{df}=63, p=0.003)$ and lower $\mathrm{FA}$ within the superior longitudinal fasciculus $(\mathrm{F}=3.02, \mathrm{df}=46$, $p=004)$ and inferior longitudinal fasciculus $(t=2.34, \mathrm{df}=46$, $p=0.023)$ in the older group.

\section{DISCUSSION}

Our data provide evidence for lower FA within the inferior longitudinal fasciculus and superior longitudinal fasciculus in both younger and older patients compared with their respective healthy volunteer groups with comparable effect sizes, suggesting that such abnormalities may reflect a traitrelated manifestation of psychosis. Moreover, our data argue against accelerated aging in psychosis from adolescence through middle adulthood. Strengths of the current study include the use of large sample sizes, young patients studied in their first episode of illness prior to extensive pharmacological intervention, and investigation of multiple indices of putative white matter microstructure.

Using manually placed ROIs, Friedman et al (2008) reported significant or trend-level lower FA in chronic patients compared with matched healthy controls in the forceps major/minor, inferior longitudinal fasciculus, and the genu/splenium of the corpus callosum. In addition, they reported lower FA within the inferior longitudinal fasciculus in their first-episode cohort compared with age- and sexmatched healthy volunteers. Compared with our younger (first episode) cohort, the patients from the first-episode cohort of Friedman et al (2008) were, on average, 5 years older, which may be due to their use of patients within 3 years of illness onset. The mean age of their 'chronic' group was nearly identical to the mean age of the older patient group used in the current study. Notably, the effect sizes for the comparison of their first-episode and chronic patient cohorts to their matched healthy volunteer cohorts were

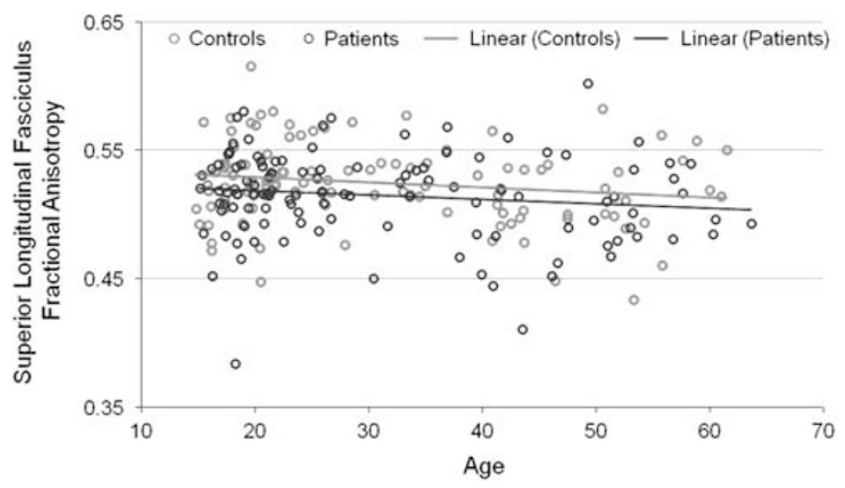

Figure 3 Scatterplot of age with fractional anisotropy in the superior longitudinal fasciculus. Note: The interactions of age and age $^{2}$ (not illustrated) with group were not statistically significant.

highly comparable ( -0.50 and -0.65 , respectively) for the inferior longitudinal fasciculus. Similarly, effect size measures (eta-squared) in the current study for this tract were comparable between the younger and older patient cohorts compared with their respective healthy volunteer groups.

In another study, Voineskos et al (2010) investigated white matter tracts in 25 younger patients with schizophrenia $(\leqslant 55$ years), 25 younger controls, 25 older patients with schizophrenia ( $\geqslant 56$ years), and 25 older controls. These authors reported lower FA in the left uncinate fasciculus and right cingulum bundle in younger patients compared with matched controls. It should be noted that their cohort of young patients with schizophrenia (mean age $=40$ years) was comparable to our cohort of older patients with schizophrenia (mean age $=45.5$ years), and in that regard, group differences in putative white matter microstructure were observed in cohorts of comparable age, albeit in different regions. Differences in regional specificity between our cohort and Voineskos et al (2010) may relate to sampling differences and methodology (eg, deterministic $v s$ probabilistic tractography). Similar to the current study, they did not observe age-associated differences in their patient cohort, which they attributed to 'resilience' among the older community-dwelling patients.

We did not find any evidence for aging effects on white matter measures either overall across all the tracts or specifically within tracts that differed significantly between patients and healthy volunteers. Two prior studies reported significant age-by-group interactions for whole-brain FA consistent with accelerated age-related differences in patients with schizophrenia compared with healthy volunteers (Kochunov et al, 2013; Wright et al, 2014). Differences between our study and prior studies may relate to sampling differences and our investigation of aging effects within discrete white matter tracts (in contrast to white matter across the entire brain). Furthermore, differences in illness severity among patients at the older age range, including the use of deficit patients (Voineskos et al, 2013), could conceivably influence age-associated differences in FA across the age span, especially given prior work indicating that lower FA across the schizophrenia spectrum was associated with greater symptom severity (Lener et al, 2015).

Our study also highlights the role of sex in mediating FA differences between patients and healthy volunteers. Overall, 
female patients demonstrated lower FA across the white matter tracts in the absence of significant group-by-tract-bysex interactions. Kunimatsu et al (2012) reported that male patients demonstrated higher apparent diffusion coefficient (ADC) values in the left anterior cingulum, the bilateral cingulum, and the bilateral uncinate fasciculus, whereas female patients demonstrated higher ADC values in the right anterior cingulum, the left fornix, and the bilateral uncinate fasciculus. Moreover, age-associated sex differences were comparable in both patients and healthy volunteers arguing against accelerated aging effects in psychosis.

The finding of lower FA within the inferior longitudinal fasciculus in two independent cohorts of patients strongly implicates disruption of this tract in the neurobiology of psychosis. The inferior longitudinal fasciculus is a major white matter tract that runs from the temporal to the occipital cortices above the optic radiation fibers and ventrally below the uncinate fasciculus without well-defined functions. Disruption of this white matter tract has been implicated in thought disorders, visual emotion, and cognitive impairment (Chanraud et al, 2010), which have been reported in patients with psychosis, and especially individuals with visual and auditory hallucinations (Ćurčić-Blake et al, 2015). A recent meta-analysis (Yao et al, 2013) reported disruption of the inferior longitudinal fasciculus in first-episode schizophrenia and has been identified as a potential biomarker of vulnerability to developing psychosis among early-onset patients (Epstein et al, 2014).

We also identified abnormalities within the superior longitudinal fasciculus in patients with psychosis. This tract runs dorsally along an anterior-posterior course and has been implicated in the regulation of motor behavior (Tseng et al, 2013) and transfer of somatosensory information, including language articulation (Sarubbo et al, 2015). White matter abnormalities in the superior longitudinal fasciculus have been reported both in schizophrenia (Karlsgodt et al, 2008) and psychotic bipolar disorder (Whalley et al, 2013) and are particularly robust in the early stages of psychosis (Samartzis et al, 2014). Moreover, abnormalities in this tract and its connections have been identified among individuals at risk for developing psychotic disorders (Karlsgodt et al, 2009), which predict neuropsychological and social/role functioning (Karlsgodt et al, 2008).

There are several limitations to our study. The crosssectional nature of our study precludes firm conclusions regarding the trajectory of these differences that might otherwise be obtained through longitudinal designs. We could not disentangle the effects of illness progression that may occur in poor outcome patients and the potential effects of antipsychotic treatment (Szeszko et al, 2014). Nevertheless, comparable effect sizes between younger and older patient groups argue against both possibilities. Moreover, the age range of the younger and older cohorts differed, which could conceivably bias the trajectory of the slopes. There is also the possibility of a cohort effect such that only a subgroup of younger patients may progress to become included in studies of older patients, and thus the latter group may not be representative of the former. It should also be acknowledged that accelerated aging in psychosis may be a heterogeneous process. We note taking the average of all voxels belonging to a tract in contrast to those with the highest FA in the middle of a tract (as is carried out in tract-based spatial statistics) as a potential study limitation and source of difference in findings from other studies. For example, Kochunov et al (2013) used tract-based spatial statistics to compute population average FA values along the 12 white matter tracts. Additional study limitations include possible substance use and/or taking medications other than antipsychotics at the time of the scan and the possible inclusion of individuals with anxiety and/or personality disorders, diabetes, and/or hypertension. Finally, we did not correct for motion induced by the cardiac cycle.

In sum, we provide a comprehensive investigation of multiple indices of putative white matter microstructure for nine white matter tracts in younger and older patients with psychosis from adolescence to middle adulthood. FA was lower in the inferior longitudinal fasciculus and superior longitudinal fasciculus in both patient cohorts compared with matched healthy volunteers. Our findings further argue against accelerated aging in the neurobiology of psychosis.

\section{FUNDING AND DISCLOSURE}

This work was supported in part by grants from the Brain and Behavior Research Foundation (to Dr Szeszko) and the National Institute of Mental Health to Dr Szeszko (MH76995) and Dr Malhotra (MH079800), the NSLIJ Research Institute General Clinical Research Center (M01 RR18535), an Advanced Center for Intervention and Services Research (MH74543), and a Center for Intervention Development and Applied Research (MH80173). Dr Malhotra serves on the advisory boards of Genomind, LLC and FORUM Pharmaceuticals. Dr Robinson has been a consultant to Asubio and Otsuka, and he has received grants from Bristol Meyers Squibb, Janssen, and Otsuka. The other authors declare no conflict of interest.

\section{ACKNOWLEDGMENTS}

We thank the individuals who participated in this study and Michelle Bergman and Jamie Wagner for study coordination.

\section{REFERENCES}

Abel KM, Drake R, Goldstein JM (2010). Sex differences in schizophrenia. Int Rev Psychiatry 22: 417-428.

Behrens TEJ, Woolrich MW, Jenkinson M, Johansen-Berg $\mathrm{H}$, Nunes RG, Clare $S$ et al (2003). Characterization and propagation of uncertainty in diffusion-weighted MR imaging. Mag Reson Med 50: 1077-1088.

Buchsbaum MS, Tang CY, Peled S, Gudbjartsson H, Lu D, Hazlett EA et al (1998). MRI white matter diffusion anisotropy and PET metabolic rate in schizophrenia. Neuroreport 9: 425-430.

Chanraud S, Zahr N, Sullivan EV, Pfefferbaum A (2010). MR diffusion tensor imaging: a window into white matter integrity of the working brain. Neuropsychol Rev 20: 209-225.

Cheung V, Cheung C, McAlonan GM, Deng Y, Wong JG, Yip L et al (2008). A diffusion tensor imaging study of structural dysconnectivity in never-medicated, first-episode schizophrenia. Psychol Med 38: 877-886.

Ćurčić-Blake B, Nanetti L, van der Meer L, Cerliani L, Renken R, Pijnenborg GH et al (2015). Not on speaking terms: hallucinations and structural network disconnectivity in schizophrenia. Brain Struct Funct 220: 1-12. 
Ellison-Wright I, Bullmore E (2009). Meta-analysis of diffusion tensor imaging studies in schizophrenia. Schizophr Res 108: 3-10.

Epstein KA, Cullen KR, Mueller BA, Robinson P, Lee S, Kumra S (2014). White matter abnormalities and cognitive impairment in early-onset schizophrenia-spectrum disorders. J Am Acad Child Adolesc Psychiatry 53: 362-372.

Fitzsimmons J, Kubicki M, Shenton ME (2013). Review of functional and anatomical brain connectivity findings in schizophrenia. Curr Opin Psychiatry 26: 172-187.

Friedman J, Tang C, Carpenter D, Buchsbaum M, Schmeidler J, Flanagan L et al (2008). Diffusion tensor imaging findings in firstepisode and chronic schizophrenia patients. Am J Psychiatry 165: 1024-1032.

Gogos A, Kwek P, van den Buuse M (2012). The role of estrogen and testosterone in female rats in behavioral models of relevance to schizophrenia. Psychopharmacology 219: 213-224.

Goldstein JM, Cherkerzian S, Tsuang MT, Petryshen TL (2013). Sex differences in the genetic risk for schizophrenia: history of the evidence for sex-specific and sex-dependent effects. Am J Med Genet B Neuropsychiatr Genet 162B: 698-710.

Jenkinson M, Beckmann CF, Behrens TE, Woolrich MW, Smith SM (2012). FSL. Neuroimage 62: 782-790.

Karlsgodt KH, Sanz J, van Erp TG, Bearden CE, Nuechterlein KH, Cannon TD (2009). Re-evaluating dorsolateral prefrontal cortex activation during working memory in schizophrenia. Schizophr Res 108: 143.

Karlsgodt KH, van Erp TG, Poldrack RA, Bearden CE, Nuechterlein KH, Cannon TD (2008). Diffusion tensor imaging of the superior longitudinal fasciculus and working memory in recent-onset schizophrenia. Biol Psychiatry 63: 512-518.

Kochunov P, Glahn DC, Rowland LM, Olvera RL, Winkler A, Yang YH et al (2013). Testing the hypothesis of accelerated cerebral white matter aging in schizophrenia and major depression. Biol Psychiatry 73: 482-491.

Kubicki M, Khan U, Westin CF, Pieper S, McCarley R, Shenton M (2008). Atlas-based white matter segmentation of DTI in schizophrenia. Schizophr Res 102: 82.

Kunimatsu N, Aoki S, Kunimatsu A, Abe O, Yamada H, Masutani Y et al (2012). Tract-specific analysis of white matter integrity disruption in schizophrenia. Psychiatry Res 201: 136-143.

Lei W, Li M, Deng W, Zhou Y, Ma X, Wang Q et al (2015). Sexspecific patterns of aberrant brain function in first-episode treatment-naïve patients with schizophrenia. Int J Mol Sci 16: $16125-16143$.

Lener MS, Wong E, Tang CY, Byne W, Goldstein KE, Blair NJ et al (2015). White matter abnormalities in schizophrenia and schizotypal personality disorder. Schizophr Bull 41: 300-310.

Lim KO, Hedehus M, Moseley M, de Crespigny A, Sullivan EV, Pfefferbaum A (1999). Compromised white matter tract integrity in schizophrenia inferred from diffusion tensor imaging. Arch Gen Psychiatry 56: 367-374.

Peters BD, Karlsgodt KH (2014). White matter development in the early stages of psychosis. Schizophr Res 161: 61-69.

Peters BD, Ikuta T, DeRosse P, John M, Burdick KE, Gruner P et al (2014). Age-related differences in white matter tract microstructure are associated with cognitive performance from childhood to adulthood. Biol Psychiatry 75: 248-256.

Rosenberger G, Kubicki M, Nestor PG, Connor E, Bushell GB, Markant D et al (2008). Age-related deficits in fronto-temporal connections in schizophrenia: a diffusion tensor imaging study. Schizophr Res 102: 181-188.

Savadjiev P, Whitford TJ, Hough ME, Clemm von Hohenberg C, Bouix S, Westin CF et al (2014). Sexually dimorphic white matter geometry abnormalities in adolescent onset schizophrenia. Cereb Cortex. 24: 1389-1396.

Sarubbo S, De Benedictis A, Merler S, Mandonnet E, Balbi S, Granieri E et al (2015). Towards a functional atlas of human white matter. Hum Brain Mapp 36: 3117-3136.

Samartzis L, Dima D, Fusar-Poli P, Kyriakopoulos M (2014). White matter alterations in early stages of schizophrenia: a systematic review of diffusion tensor imaging studies. J Neuroimaging 24: 101-110.

Schneiderman JS, Hazlett EA, Chu KW, Zhang J, Goodman CR, Newmark RE et al (2011). Brodmann area analysis of white matter anisotropy and age in schizophrenia. Schizophr Res 130: $57-67$.

Szeszko PR, Strous RD, Goldman RS, Ashtari M, Knuth KH, Lieberman JA et al (2002). Neuropsychological correlates of hippocampal volumes in patients experiencing a first episode of schizophrenia. Am J Psychiatry 159: 217-226.

Szeszko PR, Ardekani BA, Ashtari M, Kumra S, Robinson DG, Sevy $S$ et al (2005). White matter abnormalities in first-episode schizophrenia or schizoaffective disorder: a diffusion tensor imaging study. Am J Psychiatry 162: 602-605.

Szeszko PR, Robinson DG, Ikuta T, Peters BD, Gallego JA, Kane J et al (2014). White matter changes associated with antipsychotic treatment in first-episode psychosis. Neuropsychopharmacology 39: 1324-1331.

Paus T, Toro R (2009). Could sex differences in white matter be explained by g ratio? Front Neuroanat 3: 14.

Trzesniak C, Kempton MJ, Busatto GF, de Oliveira IR, Galvão-de Almeida A, Kambeitz J et al (2011). Adhesio interthalamica alterations in schizophrenia spectrum disorders: a systematic review and meta-analysis. Prog Neuropsychopharmacol Biol Psychiatry 35: 877-886.

Tseng BY, Gundapuneedi T, Khan MA, Diaz-Arrastia R, Levine BD, $\mathrm{Lu} \mathrm{H}$ et al (2013). White matter integrity in physically fit older adults. Neuroimage 82: 510-516.

Voineskos AN, Foussias G, Lerch J, Felsky D, Remington G, Rajji TK et al (2013). Neuroimaging evidence for the deficit subtype of schizophrenia. JAMA Psychiatry 70: 472-480.

Voineskos AN, Lobaugh NJ, Bouix S, Rajji TK, Miranda D, Kennedy JL et al (2010). Diffusion tensor tractography findings in schizophrenia across the adult lifespan. Brain 133: 1494-1504.

Whalley HC, Sprooten E, Hackett S, Hall L, Blackwood DH, Glahn DC et al (2013). Polygenic risk and white matter integrity in individuals at high risk of mood disorder. Biol Psychiatry 74: 280-286.

White T, Magnotta VA, Bockholt HJ, Williams S, Wallace S, Ehrlich S et al (2011). Global white matter abnormalities in schizophrenia: a multisite diffusion tensor imaging study. Schizophr Bull 37: 222-232.

White T, Nelson M, Lim KO (2008). Diffusion tensor imaging in psychiatric disorders. Top Magn Reson Imaging 19: 97-109.

Wright SN, Kochunov P, Chiappelli J, McMahon RP, Muellerklein F, Wijtenburg SA et al (2014). Accelerated white matter aging in schizophrenia: role of white matter blood perfusion. Neurobiol Aging 35: 2411-2418.

Wu YC, Hill RA, Gogos A, Van Den Buuse M (2013). Sex differences and the role of estrogen in animal models of schizophrenia: interaction with BDNF. Neuroscience 239: 67-83.

Yao L, Lui S, Liao Y, Du MY, Hu N, Thomas JA et al (2013). White matter deficits in first episode schizophrenia: an activation likelihood estimation meta-analysis. Prog Neuropsychopharmacol Biol Psychiatry 45: 100-106. 